\title{
Spatially Resolved Dynamic Eigenmode Spectrum of Co Rings
}

\author{
I. Neudecker, ${ }^{1}$ M. Kläui, ${ }^{2}$ K. Perzlmaier, ${ }^{1}$ D. Backes,${ }^{2,3}$ L. J. Heyderman, ${ }^{3}$ C. A. F. Vaz, ${ }^{4}$ \\ J. A. C. Bland ${ }^{4}$ U. Rüdiger, ${ }^{2}$ and C. H. Back ${ }^{1}$ \\ ${ }^{1}$ Institut für Experimentelle und Angewandte Physik, Universität Regensburg, Universitätsstrasse 31, D-93040 Regensburg, Germany \\ ${ }^{2}$ Fachbereich Physik, Universität Konstanz, Universitätsstrasse 10, D-78457 Konstanz, Germany \\ ${ }^{3}$ Paul Scherrer Institut, CH-5232 Villigen PSI, Switzerland \\ ${ }^{4}$ Cavendish Laboratory, University of Cambridge, Madingley Road, Cambridge, CB3 OHE, United Kingdom
}

(Received 27 September 2005; published 7 February 2006)

\begin{abstract}
The spatially resolved eigenmode spectrum of micrometer-sized Co ring elements has been determined by means of combined vector network analyzer ferromagnetic resonance and time resolved magneto-optic Kerr effect measurements. Up to 5 resonant eigenmodes were observed in the frequency range from $45 \mathrm{MHz}$ to $20 \mathrm{GHz}$ as a function of an external magnetic bias field. A well-defined mode structure was found for the two equilibrium states (vortex and onion) which correspond to distinctive spatial modes. The effect of dynamic inter-ring coupling on the modes in the remanent states was evinced. The experimental results are found to be in good agreement with those of micromagnetic simulations. Our results demonstrate that, in analogy to the well-defined static equilibrium magnetic states of ring elements, the eigenmode spectra of this high symmetry geometry consist of a well-defined and simple mode structure.
\end{abstract}

PACS numbers: 75.75.+a, 75.30.Ds, 76.50.+g

The ability to understand and engineer the eigenmode spectrum by reducing the dimensions of a system constitutes a formidable challenge in nanomagnetism. The detailed understanding is crucial for possible applications in novel magnetic storage media and sensors [1-3]. As writing times are pushed down to the region where precessional switching occurs, the intrinsic reversal processes are influenced by the eigenmode spectrum of the element. In recent years, the very complex dynamic modal spectra of only the simplest shapes, such as isolated micron sized ferromagnetic disks, squares, and stripes have been investigated [410]. In particular, high symmetry elements such as disks have been studied, which form an out-of-plane vortex core leading to a stray field. If this vortex core is removed, a ring is obtained, a geometry which has attracted much attention recently [11-14]. In the flux-closure vortex configuration, rings have no stray field and therefore would not influence neighboring elements, which might prove useful for high density storage media [2]. In addition to the flux-closure vortex state another state was found, termed the "onion state," which is characterized by two head-to-head domain walls and attained reversibly from saturation [11]. For rings, the static properties have been the most studied $[11,14,15]$, but for applications, where fast switching is crucial, the dynamic properties are of paramount interest. Furthermore, rings have an extra degree of freedom (the ring width) compared to topologically simply connected elements, such as disks. This allows for adjusting both the static and dynamic properties (which are expected to be distinctly different from the conventionally studied geometries) more precisely for a specific application.

First dynamic experiments on ring structures were recently carried out by Giesen et al. [16,17] using vector network analyzer ferromagnetic resonance (VNA-FMR). This novel inductive technique gives an insight into the modal spectrum with respect to the frequency and the effective damping of the various modes. However, it does not reveal the spatial structure of the modes, which is essential for a detailed understanding. Zhu et al. [18] carried out the first experiments on ring structures using time resolved magneto optical Kerr effect (TRMOKE) by exciting the rings with a field pulse and then observing the time evolution of the magnetization. This technique probes a superposition of some eigenmodes but does not reveal the nature of the separate eigenmodes. In order to understand and image each eigenmode, a combination of both measurement methods can be employed, leading to deeper insights into the dynamic behavior.

In this Letter we report the results of combined VNAFMR and TRMOKE measurements to obtain the resonance frequencies of the eigenmodes as a function of an external static magnetic field and to reveal the spatial structure of the corresponding eigenmodes in both the onion and vortex states. The observed modes were compared to micromagnetic simulations carried out using the LLG code [19]. By measuring rings with different edge-to-edge spacings, the dynamic dipolar coupling was probed.

For the measurements, we have employed a spatially resolved ferromagnetic resonance technique using magneto-optical Kerr effect combined with continuous wave (cw) excitation (SR-FMR-MOKE) [20]. Details concerning the setup will be published elsewhere. The spatially resolved ferromagnetic resonance was realized by modifying a TRMOKE setup [21,22]. Instead of applying a short magnetic field pulse and stroboscopically recording the magnetic response, in SR-FMR-MOKE the magnetic system is excited by means of a cw high frequency (hf) field in the plane of the rings generated by a hf current through a coplanar waveguide. In contrast to the previously used standard pulsed excitation $[6,23]$, which activates a 
complicated superposition of modes, we selectively excite only a certain eigenmode of the magnetic system in the frequency domain and simultaneously image the structure of this mode with the high spatial resolution of our Kerr setup of $\sim 300 \mathrm{~nm}$. For each eigenmode the resulting contrast reveals the spatially resolved amplitude of the magnetic excitation at a given phase.

The coplanar waveguide was fabricated on highresistivity GaAs using optical lithography, thermal evaporation and a subsequent lift-off process. On top of the center conductor in direct contact with the waveguide (10 $\mu \mathrm{m}$ wide), an array of $2 \times 350$ rings was produced by means of electron beam lithography and a lift-off technique [24]. The Co rings were grown by molecular-beam epitaxy in ultrahigh vacuum (base pressure $3 \times$ $10^{-10}$ mbar) and capped with $4 \mathrm{~nm} \mathrm{Au}$ for protection. The thickness of the Co rings was $32 \mathrm{~nm}$, their inner diameter $1.7 \mu \mathrm{m}$, and their width $500 \mathrm{~nm}$. An inter-ring spacing of $100 \mathrm{~nm}$ along the waveguide and $900 \mathrm{~nm}$ in transverse direction was chosen.

Micromagnetic simulations were carried out using the LLG Micromagnetics Simulator [19]. Edge corrections were applied both to the inner and the outer boundaries of the ring. In order to compare experimental to simulated data, the time domain output from simulations was transformed into the frequency domain by performing a phasesensitive Fourier transform procedure described in detail in Ref. [23]. This means that amplitude and phase were obtained as a function of the position on the structure at the corresponding frequencies. After combining phase and amplitude information the simulated data were directly compared to the experimental data obtained from SRFMR-MOKE.

First, inductive VNA-FMR measurements were carried out in order to reveal the frequencies of the eigenmodes as a function of the external bias field. Figure 1(b) shows data extracted from VNA-FMR with 5 distinct resonance lines at different frequencies measured at $\mu_{0} H_{\text {bias }}=80 \mathrm{mT}$ applied along the waveguide. The frequencies of the modes were extracted by fitting the peaks to Lorentzian line shapes. By performing this procedure as a function of the bias field we obtained Fig. 1(a) which shows the absorption spectrum of the rings for an up-sweep (sweeping from negative to positive bias fields). The VNA-FMR data shows a double switching process [11] from onion to vortex to reverse onion states. As a consequence we observe an asymmetry in the frequency versus field data; see Fig. 1(a). A similar behavior was observed by Giesen et al. [16]. At zero bias field one single mode (mode 1) was observed at a frequency of $f=12.9 \mathrm{GHz}$. A second mode (mode 2) appears at $\mu_{0} H_{\text {bias }}=40 \mathrm{mT}$ and $f=14 \mathrm{GHz}$, a third one (mode 3) at $\mu_{0} H_{\text {bias }}=61 \mathrm{mT}$ and $f=$ $14.1 \mathrm{GHz}$, and a fourth one (mode 4$)$ at $\mu_{0} H_{\text {bias }}=$ $80 \mathrm{mT}$ and $f=14.1 \mathrm{GHz}$. Additionally a mode that lies clearly lower in frequency (mode 5) appears at $\mu_{0} H_{\text {bias }}=$ $51 \mathrm{mT}$ and $f=6.2 \mathrm{GHz}$.

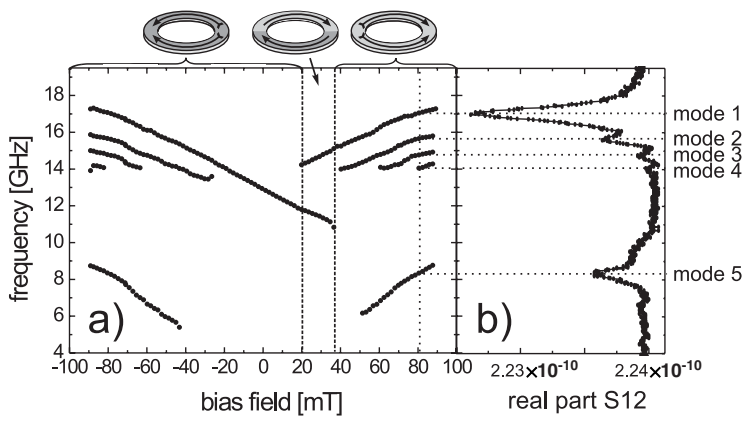

FIG. 1. (a) Frequencies (dots) of the various modes as a function of the magnetic bias field for an up-sweep obtained by inductive VNA-FMR measurements. The region of vortex state is enclosed by the vertical dashed lines in (a). The schematic drawings on top depict both onion and vortex states. (b) Raw data of the various modes at a field of $\mu_{0} H_{\text {bias }}=$ $80 \mathrm{mT}$. The resonance peaks discussed in the text are labeled from 1 to 5 .

The crucial step is now the determination of the spatial structure of the eigenmodes of the two remanent states. Mode 1 was investigated by means of SR-FMR-MOKE [see Fig. 2(a)]. Approaching zero field coming from negative bias fields, the fundamental mode of the remanent onion state was observed as shown in Fig. 2(a), left panel $(f=12.9 \mathrm{GHz})$. It shows the same symmetry as the onion configuration relative to the $x$ axis (along the waveguide). By increasing the bias field up to $\mu_{0} H_{\text {bias }}=29 \mathrm{mT}$ the rings switch into the vortex state. In agreement with VNAFMR measurements two resonances at different frequencies were found by SR-FMR-MOKE; see Fig. 2(a), middle panels $(f=14.6 \mathrm{GHz}$ and $f=11.6 \mathrm{GHz})$. The spatially resolved images show that the two modes are located in opposite "arms" of the rings. The reason for this behavior is the influence of the bias field on the effective field inside the rings. In the arm magnetized parallel to the bias field the effective field is raised, whereas in the arm magnetized antiparallel it is lowered causing higher and lower resonance frequencies, respectively. Returning the field from $29 \mathrm{mT}$ back to zero bias field (minor loop), both regions show resonance at the same frequency; see Fig. 2(a), right panel $(f=12.9 \mathrm{GHz})$ which corresponds to the fundamental mode of the remanent vortex configuration. Data obtained from micromagnetic simulations support this interpretation of the experimental results.

In order to reveal the structure of the additional modes found by VNA-FMR at bias fields above $\mu_{0} H_{\text {bias }}=$ $40 \mathrm{mT}$, SR-FMR-MOKE measurements were carried out at $\mu_{0} H_{\text {bias }}=80 \mathrm{mT}$. The corresponding images (labeled E) are shown together with data obtained by micromagnetic simulations (labeled $S$ ) in Fig. 2(b) (mode 1-mode 5). In order to extract the modal structure from the images as a function of the azimuthal angle $(\varphi)$ angular scans averaged over the hole width of the ring were performed for the various modes. The experimental and simulated data are shown for $0^{\circ} \leq \varphi \leq 180^{\circ}$, and 
a) $\mu_{0} H_{\text {bias }}=0 \mathrm{mT}, \quad \mu_{0} H_{\text {bies }}=29 \mathrm{mT}, \quad \mu_{0} H_{\text {bas }}=29 \mathrm{mT}, \quad \mu_{0} H_{\text {daas }}=0 \mathrm{mT}$, $f=12.9 \mathrm{GHz} \quad f=14.6 \mathrm{GHz} \quad f=11.6 \mathrm{GHz} \quad f=12.9 \mathrm{GHz}$ (6)

b)

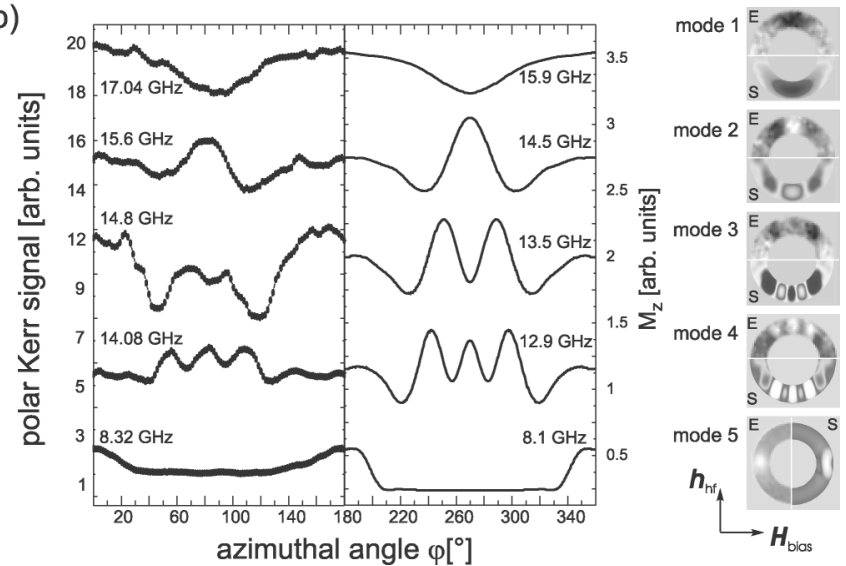

FIG. 2. The images show the local Kerr rotation strength obtained from experimental SR-FMR-MOKE investigations (labeled by E) together with the data from micromagnetic simulations (labeled by $\mathrm{S}$ ). Black and white representing the amplitude have a phase difference of $180^{\circ}$. Figure (a) shows mode 1 during the reversal process for an up-sweep. The schematic drawings indicate the corresponding static magnetization configuration. Figure (b) shows the various modes at $\mu_{0} H_{\text {bias }}=80 \mathrm{mT}$. Angular scans along the ring perimeter provide the Kerr signal as a function of the azimuthal angle $\varphi$ obtained by experiment $\left(0^{\circ} \leq \varphi \leq 180^{\circ}\right)$ and by simulations $\left(180^{\circ} \leq \varphi \leq 360^{\circ}\right)$ as shown in the graph.

$180^{\circ} \leq \varphi \leq 360^{\circ}$, respectively. For modes 1 to 4 , the symmetry of the underlying onion state is retained, meaning that the modes are symmetric with respect to the horizontal axis (parallel to the field through the ring center). These modes increase the number of azimuthal nodal lines from one (regarding one half of the ring) for mode 1 always by two for the next higher mode. The discrepancy between experiment and simulation in the frequency is less than $9 \%$ and arises probably from the uncertainty of the sample thickness.

In the following we assign wave numbers $k_{\varphi}=1 / \lambda$ for the various modes extracted from the angular scans in Fig. 2(b). The resulting dispersion relation for the experimental and for the simulated data is shown in Fig. 3. Due to the decrease in frequency with increasing wave number, and since the wave vectors $\boldsymbol{k}$ are approximately parallel to the magnetization $\boldsymbol{M}$, these modes are reminiscent of magnetostatic backward-volume modes [25].

For a better understanding of the nature of the observed modes the static spin configuration was extracted from the micromagnetic simulations. Figure 4(a) shows the static spin configuration represented by the arrows together with the $y$ component of the magnetization $\boldsymbol{M}$ encoded by the black-white contrast. The dynamic modal structure of mode 1 and mode 5 (grayscale) is shown together with the static magnetization configuration (arrows) in Figs. 4(b)

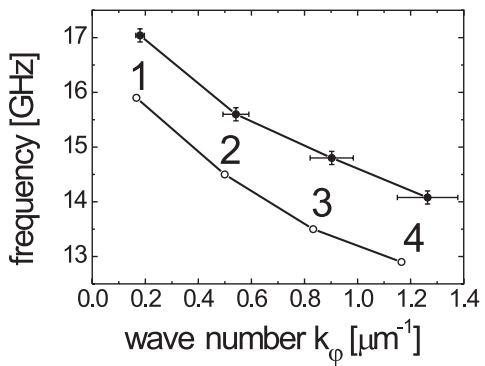

FIG. 3. The frequencies of the four highest modes as a function of their wave numbers at $\mu_{0} H_{\text {bias }}=80 \mathrm{mT}$ obtained by experiment (solid dots) and by simulation (open circles). The lines are guides to the eye. The numbers label the different modes.

and 4(c), respectively. It was found that modes 1 to 4 are located in the regions where the magnetization is approximately parallel with respect to the bias field and the ring edges [see Figs. 4(a) and 4(b)]. Figures 4(a) and 4(c) reveal that mode 5 is localized in the transverse domain wall $[5,26]$ of the onion state, where the magnetization is also aligned parallel to the external magnetic field but perpendicular to the edges of the rings. As a consequence the total effective field in this area is lowered with respect to the arms leading to the lower resonance frequency of this localized mode.

At this point it should be noted that modes 1 to 4 , namely, the modes with the magnetization parallel to the ring edges, show an odd number of nodes only. We are not able to detect modes with an even number of nodes in either the experiment or the simulation. This is expected from our uniform excitation geometry. The quasi-uniform magnetization within the arms of the rings is excited by a uniform hf field. In this case the selection rule for excitation implies that the number of half wavelengths should be odd, as has already been stated by Kittel in Ref. [27]. We note that nevertheless the modes with an even number of nodes should be detectable in Brillouin light scattering experiments.
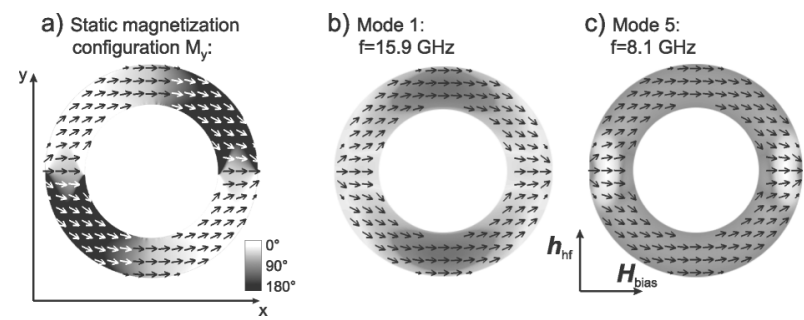

FIG. 4. Simulation of the static magnetization configuration together with the dynamic modal structure at $\mu_{0} H_{\text {bias }}=80 \mathrm{mT}$. The arrows indicate the direction of the magnetization. In (a) the static $y$ component of the magnetization $\boldsymbol{M}$ is represented by the black-white contrast (negative and positive $y$ directions). Panels (b) and (c) show the dynamic modes 1 and 5 superimposed by the static spin configuration, respectively. It can be seen that these modes are localized at the arms of the ring (b) and the domain wall area (c). 


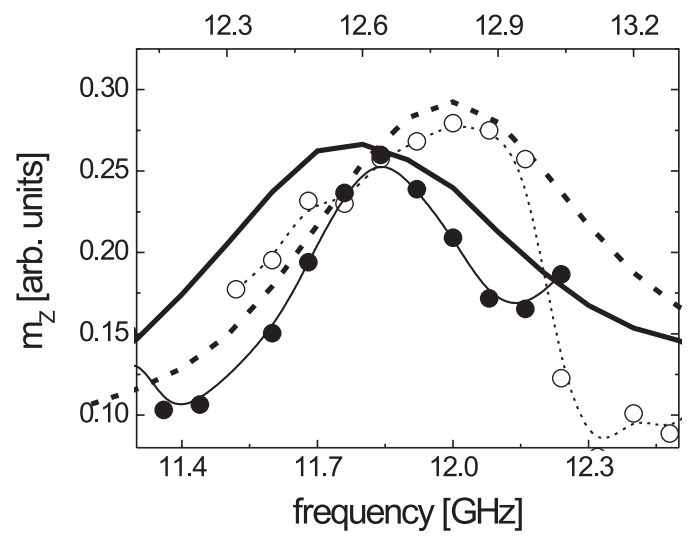

FIG. 5. The global Fourier transform of simulated (lines) data together with experimental data from frequency sweeps using SR-FMR-MOKE (dots and lines) for the remanent onion state at $\mu_{0} H_{\text {bias }}=0 \mathrm{mT}$. Simulated and experimental data correspond to the lower and upper frequency axis, respectively. The solid and the dashed lines represent the single and the coupled ring, respectively.

Because of the small spacing of the rings along the $x$ direction, static coupling in an applied field was observed by means of MOKE loops. We qualitatively observe the same behavior as previously reported [28], namely, a narrowing of the hysteresis loop of approximately $2 \mathrm{mT}$ for small inter-ring spacing. In order to investigate the effect of coupling on the dynamic properties, we performed frequency sweeps for different spaced rings using SR-FMRMOKE. After finishing the measurements described in the previous sections some rings on the array were removed by means of a focused ion beam. As a result single rings with inter-ring spacing greater than $900 \mathrm{~nm}$ could be investigated. We found that, as expected, the frequencies of the mode in the onion state are shifted by $\Delta f \approx 160 \mathrm{MHz}$ (see Fig. 5). To confirm this result, simulations were carried out for a single ring and for a row of rings using periodic boundary conditions, where we found a frequency shift of $\Delta f=200 \mathrm{MHz}$ for the fundamental modes in the onion state and in the vortex state (see Fig. 5). The fact that the frequency shift is the same for the modes in the onion and the nominally stray-field free vortex state implies that the origin of the dynamic coupling cannot be static. Therefore the frequency shift results from dynamic coupling, which shows that in the excited eigenmodes even the small dynamic deviations of the spins from the stray-field free static vortex state are sufficient to mediate significant coupling.

In summary, the influence of the magnetic configuration of micrometer ring magnets on the eigenmode structure was measured and the detailed eigenmode spectra for the two stable remanent states (vortex versus onion states) have been determined. We observed up to five resonant eigenmodes in the frequency range from 0.045 to $20 \mathrm{GHz}$ as a function of the applied magnetic field. Direct spectral imaging of the amplitude distribution has allowed us to determine the eigenmode structure in ferromagnetic rings for the first time. These modes correspond to four longitudinal modes, with magnetostatic backward-volume character, and a transverse mode, localized in the transverse domain wall. We also find that the effect of interactions is to increase the eigenmode frequencies, which is shown to be related to a dynamic coupling process occurring between the elements. This study demonstrates that these high symmetry structures exhibit simple eigenmode behavior and that frequency dependence measurements are an ideal probe of dynamic spin interactions.

We would like to thank Georg Woltersdorf for stimulating discussions. The use of the FIB at IBM Rueschlikon is gratefully acknowledged. Furthermore, financial support by the DFG through the Priority Programme 1133 "Ultrathin Fast Magnetization Processes," the SFB 513, the EPSRC (U.K.), and the CMI Magnetoelectronic Devices is gratefully acknowledged.

[1] G. A. Prinz, J. Magn. Magn. Mater. 200, 57 (1999).

[2] J.-G. Zhu et al., J. Appl. Phys. 87, 6668 (2000).

[3] M. M. Miller et al., Appl. Phys. Lett. 81, 2211 (2002).

[4] J. Jorzick et al., Phys. Rev. Lett. 88, 047204 (2002).

[5] J. P. Park et al., Phys. Rev. B 67, 020403(R) (2003).

[6] M. Buess et al., Phys. Rev. Lett. 93, 077207 (2004).

[7] L. Giovannini et al., Phys. Rev. B 70, 172404 (2004).

[8] K. Perzlmaier et al., Phys. Rev. Lett. 94, 057202 (2005).

[9] D. Chumakov et al., Phys. Rev. B 71, 014410 (2005).

[10] B. A. Ivanov and C. E. Zaspel, Phys. Rev. Lett. 94, 027205 (2005).

[11] J. Rothman et al., Phys. Rev. Lett. 86, 1098 (2001).

[12] S. P. Li et al., Phys. Rev. Lett. 86, 1102 (2001).

[13] F. J. Castano et al., Phys. Rev. B 67, 184425 (2003).

[14] M. Kläui et al., J. Phys. Condens. Matter 15, R985 (2003).

[15] T. Uhlig and J. Zweck, Phys. Rev. Lett. 93, 047203 (2004).

[16] F. Giesen et al., Appl. Phys. Lett. 86, 112510 (2005).

[17] F. Giesen et al., J. Appl. Phys. 97, 10 A712 (2005).

[18] X. Zhu et al., Appl. Phys. Lett. 86, 262502 (2005).

[19] M.R. Scheinfein, the LLG code can be found at http:// llgmicro.home.mindspring.com/.

[20] S. Tamaru et al., J. Appl. Phys. 91, 8034 (2002).

[21] Y. Acremann et al., Science 290, 492 (2000).

[22] W. K. Hiebert, A. Stankiewicz, and M. R. Freeman, Phys. Rev. Lett. 79, 1134 (1997).

[23] M. Buess et al., Phys. Rev. Lett. 94, 127205 (2005).

[24] L. J. Heyderman et al., J. Appl. Phys. 93, 10011 (2003).

[25] R. W. Damon and J. R. Eshbach, J. Phys. Chem. Solids 19, 308 (1961).

[26] J. Raabe et al., Phys. Rev. Lett. 94, 217204 (2005).

[27] C. Kittel, Phys. Rev. 110, 1295 (1958).

[28] M. Kläui et al., Appl. Phys. Lett. 86, 032504 (2005). 